\title{
Depressão e efeitos da COVID-19 em universitários
}

\author{
Gabrielli Algazal Marin'1 \\ Jullya Martins Bianchin ${ }^{1}$ \\ Igor Ruan De Araujo Caetano ${ }^{1}$ \\ Fernanda Liboni Cavicchioli ${ }^{1}$
}

\section{RESUMO}

Introdução: Em 2019 e 2020 o mundo foi afetado pela pandemia da coronavírus (COVID-19) - uma emergência de saúde pública, conforme a Organização Mundial da Saúde (OMS). A resposta ao aumento no número de infecções e mortes foi o fechamento de diversos ambientes e a imposição do distanciamento social, fato que alterou os locais de interação e vivência dos estudantes universitários.

Objetivos: Evidenciar e compreender os reflexos psicossociais, especialmente a depressão em virtude da doença e do cenário pandêmico. Métodos: Trata-se de revisão literária narrativa. Foram encontrados 234 artigos, na base dados PUBMED e PubMed Central com as palavras-chave "students"; "depression"; "quarantine"; "coronavirus infections". Foram excluídos artigos que não trataram de depressão, estudantes ou que não abordavam estudantes universitários ou não diferenciaram as populações no estudo, restando 14 artigos. Resultados: Foram observados diversos impactos na vida dos estudantes universitários, como também a queda no desempenho estudantil no ensino superior e o agravamento de sintomas depressivos com o confinamento, $(\mathrm{P}<0,001)$ em especial naqueles infectados $(\mathrm{P}<001)$. O maior sedentarismo, a redução da prática de exercício físico, tempo de sono, piora da dieta auxiliaram no aumento dos sintomas depressivos. O comportamento foi alterado com aumento da reclusão, percepção de risco, e ideação suicida $(P<0,001)$. Discussão: o cenário pandêmico aumentou os índices de depressão entre estudantes universitários, bem como a falta de adesão ao tratamento dos que já possuíam a doença. O confinamento, o sedentarismo, o maior número de horas em tela, de notícias pelas mídias, a piora da alimentação e a frustração dos estudantes diante da realidade acadêmica estão todos relacionados ao aumento dos quadros depressivos. São necessários novos estudos que envolvam a depressão e os estudantes universitários. Raros são os estudos longitudinais, como também, intervencionistas, apesar de ambos serem essenciais para que as intervenções pelo sistema de saúde sejam eficazes e convenientes, não apenas nesta, mas também em próximas pandemias possíveis.

Termos de indexação students, depression, quarantine e Coronavirus, Infections".

${ }^{1}$ Pontifícia Universidade Católica do Paraná, Faculdade de Medicina. Av. Jockei Club, 485, Hípica, 86072-360, Londrina, PR, Brasil. Correspondência para: FL Cavicchioli. E-mail:<liboni.fernanda@pucpr.br>. 


\section{INTRODUÇÃO}

No ano de 2020, o novo coronavírus (COVID-19) assolou o mundo e foi definida pela Organização Mundial de Saúde (OMS) como uma emergência de saúde pública $[1,2]$. As infecções e mortes, que aumentavam exponencialmente, precisaram de medidas que impedissem o seu avanço [1]. Nesse sentido, além do uso de máscaras e luvas, do hábito frequente de lavar as mãos com água e sabão ou álcool em gel, o "distanciamento social" foi aplicado para reduzir interações físicas entre as pessoas como forma de evitar a transmissão viral; eventos foram cancelados desde então, bem como, mercados, lojas, escritórios, escolas e universidades foram fechados $[1,3,4]$.

Nesse contexto, é fundamental compreender que essas diversas ações de segurança adotadas pela sociedade afetaram psicologicamente diversos grupos sociais, inclusive estudantes [4]. Aliado a isso, a preocupação, juntamente com o medo de ser infectado, agravaramse drasticamente, somados ao sentimento gerado pela tempestade de incontáveis notícias sobre a pandemia e a COVID-19, informações, as quais, nem sempre exprimiam veracidade dos fatos [4].

Na epidemia de SARS-CoV-2, também em anteriores, como a de SARS-Cov-1 e MERS-Cov-2, que compartilham semelhanças estruturais, biológicas e de porta de entrada com o COVID-19, foram identificados neurotropismo e presença do vírus no sistema nervoso central, como também no líquor cefalorraquidiano. Além disso, foram relatados distúrbios orgânicos semelhantes a insônia, depressão e agressividade em infectados. Consequentemente, tais distúrbios também foram descritos por jovens estudantes, os quais, muitas vezes não eram compreendidos nem mesmo pela própria família [2].

Não apenas isso, o impacto do atual cenário pandêmico vai muito mais além do dano cerebral direto pelo vírus. O aumento do pânico, da doença em si, a quarentena e as restrições públicas podem ter um impacto significativo na saúde mental mundial [2]. As pesquisas indicam que o isolamento, como também as mudanças obrigatórias de rotina e de comportamento geraram profundas mudanças no nível de saúde mental nos indivíduos [5-7].

Com relação a área da saúde especificamente, é bem verdade que, a administração do estresse, dos sentimentos, bem como o apoio social e a distribuição de tarefas são fatores de extrema importância para enfrentar a pandemia [7]. Contudo, as extensas cargas horárias, as altas intensidade e frequências de trabalho no período, bem como os riscos ocupacionais, exacerbaram o estresse mental, a ansiedade e depressão não apenas em profissionais, mas também em estudantes universitários que serão futuros integrantes desta área [7,8]. Além disso, os estudantes de medicina, especificamente, se mostraram especialmente fragilizados com relação a depressão, possivelmente em virtude da menor experiência em lidar com ambientes de alto risco quando comparados aos formados nessa atuação [7].

Quando se trata de estudantes da graduação em geral, as pesquisas sugerem que, no primeiro semestre letivo de 2020, eles apresentaram-se mais ansiosos e depressivos, independentemente da área de atuação. E isso é explicado pelo aumento do sedentarismo, do uso de dispositivos eletrônicos, pela interrupção da socialização e pelo crescimento exponencial de notícias com maior percepção de risco relacionados à pandemia $[2,4,8]$.

Foram relatados, inclusive, afetos negativos e sintomas de ansiedade e depressão em estudantes universitários com apenas duas semanas de confinamento, sendo que, os fatores: grau mais alto de estudo e pontuações mais altas em ansiedade e depressão prévias foram preditores do maior afeto negativo [9].

A importância desse contexto, que cursa com o aumento de estresse, ansiedade, depressão deve ser ressaltada por promover efeitos nocivos que afetam negativamente o estado emocional, qualidade do sono, comportamento e outros âmbitos de saúde mental [7]. Está claro que entender as implicações comportamentais, de saúde mental e a prevalência da depressão e da ansiedade são fundamentais para fornecer dados que sustentem ações pelas políticas públicas, bem como, para garantir o preparo dos governos em futuras pandemias [4]. Sendo assim, nota-se que as pesquisas são fundamentais para a promoção de estratégias de combate a essa doença, bem como, de proteção da saúde mental e de bem-estar geral dos futuros profissionais e integrantes do mercado de trabalho.

Contraditoriamente, existem raros estudos que trazem a realidade brasileira dos estudantes universitários no contexto da pandemia da COVID-19 e a depressão. Especialmente, mesmo no âmbito mundial, são raros os estudos longitudinais, ou de intervenção - com propostas de solução ao problema.

A partir do exposto, a presente revisão narrativa tem como objetivo o aprofundamento e a problematização dessa temática, para - não apenas - favorecer a mais assertiva compreensão dos possíveis efeitos psicossociais 
nos estudantes da graduação, como também dar enfoque a necessidade da pesquisa sobre o assunto, inclusive no Brasil - para que sirva de base a uma eficaz intervenção pelos serviços públicos que objetivam o zelo ao bem-estar e à saúde mental dos estudantes universitários em uma sociedade pós-pandemia.

\section{MÉTODOS}

Trata-se de uma revisão narrativa da literatura. A busca de artigos incluiu pesquisa nas bases eletrônicas PUBMED e PubMed Central (PUBMED). O período de abrangência foi entre o dia 01 de janeiro de 2020 até o dia 09 de setembro de 2020.

Nesse sentido, com relação às palavras-chave, utilizamos os descritores padronizados pelos Descritores em Ciências da Saúde, na língua inglesa, a serem: "students"; "depression"; "quarantine"; "coronavirus infections". Na plataforma PUBMED foram encontrados 9 resultados; desses, 5 artigos foram excluídos, pois se repetiam na plataforma PMC, restando 4 artigos encontrados no PUBMED. Enquanto no PMC, por sua vez, foram encontrados 234 artigos.

A partir desses resultados, foram aplicados os critérios de exclusão. Do total de 238 artigos, excluídos os repetidos, 93 artigos não particularizaram a população de estudantes em sua população amostral; 26 artigos não tratavam depressão; 32 artigos não especificaram se a população de estudantes era de universitários; 13 artigos abordavam o tema estudantes universitários, mas não os diferenciavam da população em geral no cálculo dos resultados na discussão e 60 artigos não tratavam de depressão e de estudante. Dessa forma, 14 estudos foram selecionados.

Esquematização dos critérios de inclusão e exclusão para a seleção dos estudos.

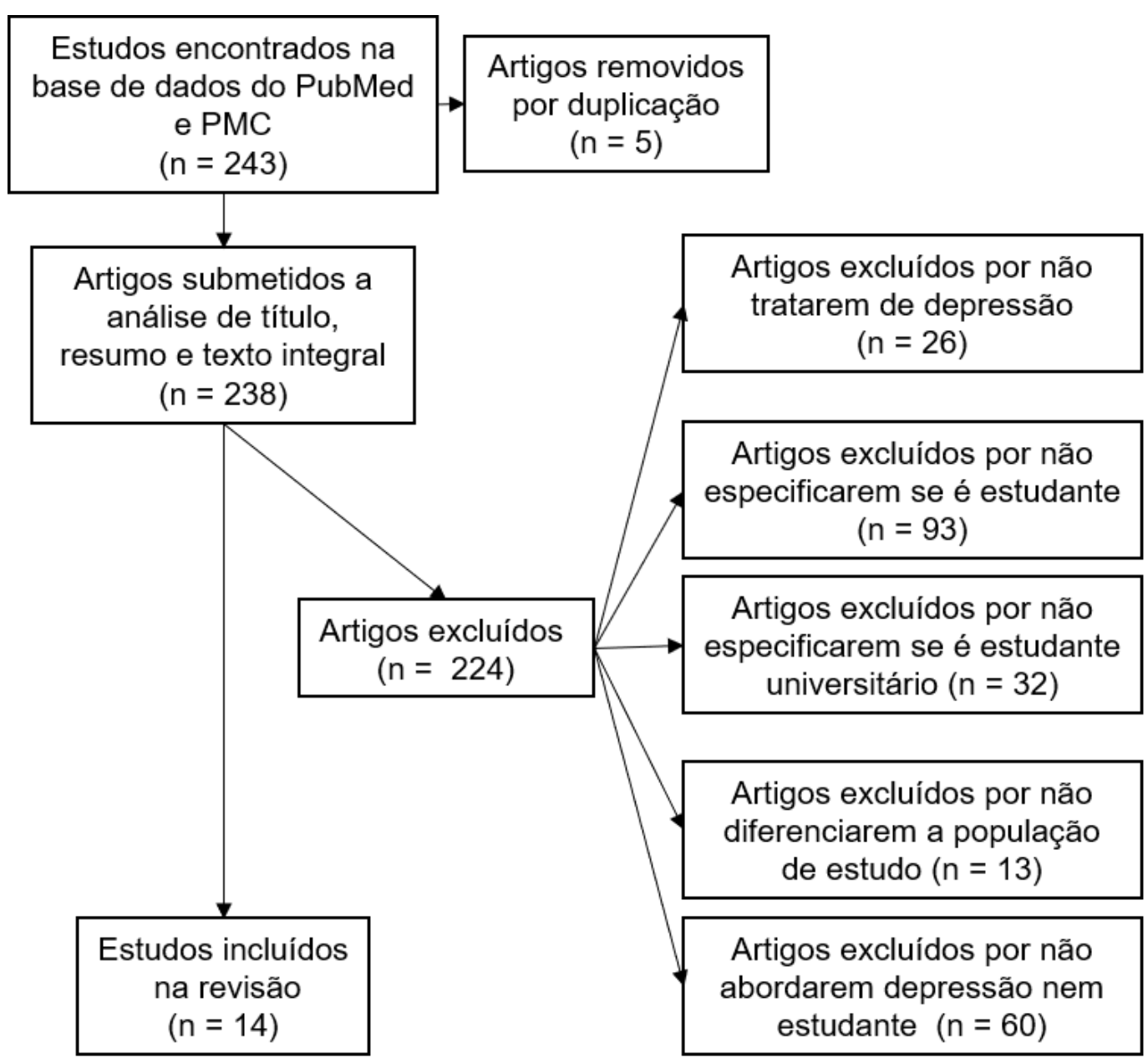

Figura 1. Elaborada pelos autores 


\section{RESULTADOS}

A seguir, serão explanados os principais fatores e impactos no estilo de vida envolvendo depressão e estudantes universitários.

\section{A realidade do estudante do ensino superior}

A pandemia atingiu diretamente os universitários em virtude de as instituições estarem temporariamente fechadas. De forma geral, tanto o adiamento de intercâmbios, cerimônias de formatura quanto o acesso a aula online foram prejudiciais do ponto de vista psicológico. Nesse sentido, estudo longe de casa e da família, o medo de não a ver novamente por período indeterminado, bem como receios pela infecção própria e de conhecidos são fatores mais preponderantes no que diz respeito a preocupação dos estudantes [2].

As populações de estudantes podem ser mais vulneráveis a ansiedade e depressão relacionados ao estresse [1]. A depressão leve é uma das principais formas de apresentação, e, apesar de ser algo presente em diversos estudos, dois devem ser destacados [5]: Estudo transversal com 119 participantes do ensino superior com prevalência de acometimento alta, e em grande maioria com graus leves de depressão conforme o Manual Diagnóstico e Estatístico-5 (DSM-5) [5]. Estudo com 476 estudantes universitários no período, com prevalência de $82,4 \%$ e sintomas depressivos que variam de leves a graves [6].

Foi encontrado também uma análise longitudinal que acompanhou anualmente a saúde mental de universitários no programa "StudentLife, com a coleta de informações de forma recorrente, portanto. Os resultados contaram com indivíduos mais deprimidos em comparação com períodos acadêmicos anteriores $(p<0,01)$ [4]. A partir da semana de suspensão das aulas e de descoberta do primeiro caso próximo ao campus a depressão permaneceu acima de níveis de outros tempos anteriores a pandemia e aumentaram ainda mais durante o período que antecede o final do período de provas finais [4]. Um estudo transversal feito com 1752 estudantes universitários e mais outros 778 membros da Universidade de Valladolid na Espanha analisou os efeitos psicológicos da pandemia e demonstrou que os níveis de depressão de estudantes eram maiores quando comparados a trabalhadores, bem como em relação a alunos do mestrado $(P<0,05)[10]$.

$O$ desempenho nas atividades curriculares exercidas pelos estudantes nesse momento também foi um fator que afetou na presença da depressão. Com uma prevalência de $76,78 \%$ dentre 476 estudantes, o atraso nas matérias
( $P=0,02)$, a diminuição da participação do acadêmico em atividades como aulas particulares $(P=0,04)$ aliada a preocupação financeira com o compromisso foram os fatores principais [6].

Em relação ao tempo de estudo no ensino superior, há controvérsias sobre se há e qual é a influência na relação com a depressão. Um estudo com 1752 estudantes concluiu que a depressão está presente em níveis mais baixos em estudantes de anos mais avançados quando comparados aos primeiros anos da graduação [10]. No entanto, outro com 555 estudantes indica que os níveis mais avançados estão relacionados ao maior nível de depressão (ambos com $\mathrm{P}<0,05$ ), e este último busca justificar a causa de os períodos mais avançados serem os mais afetados devido a grade compacta, o medo do prejuízo ao progresso e término dos estudos, bem como, outras análises apoiam essa relação esclarecendo que é devido à incerteza em relação ao futuro após a formação $[11,12]$.

Uma associação relevante é a comparação entre grupos de pacientes estudantes com e sem coronavírus. Quando os dois grupos são comparados, o resultado indica que os níveis de depressão mais altos estão entre os pacientes infectados pelo vírus respiratório $\mathrm{P}<0,001$ [5].

Por fim, um fato interessante apareceu quando grupos de estudantes, profissionais da saúde, pacientes com COVID-19 e pessoas da comunidade foram entrevistadas. $23,4 \%$ dos participantes eram estudantes de medicina, quando comparado com todos os demais grupos, fizeram a maior pontuação em ansiedade, depressão e estresse $P=0,001$ [7].

\section{Exercício físico}

O exercício físico aprimora os mecanismos de defesa do organismo, melhora a autoestima e a saúde mental, favorece satisfação, e ainda, é fundamental no fornecimento de distração - ao afastar os indivíduos de pensamentos preocupantes e negativos, especialmente em períodos de pandemia, juntamente com o acesso a cascatas de informações a todo tempo [8].

Com relação à pesquisa sobre os exercícios físicos moderados, foi observado que há impacto oposto aos exercícios físicos mais intensos e extenuantes - uma vez que, os primeiros demonstraram benefícios com relação à resposta imune inata contra possíveis infecções respiratórias, como a infecção por Sars-Cov2, enquanto as atividades extenuantes, devido ao mecanismo de aumento da ventilação alveolar, aumentam a suscetibilidade do organismo de contrair infecções das vias aéreas [8]. 
A prática de exercício físico foi associada negativamente a depressão, antes e durante a pandemia [1]. Foram relatadas dificuldades dos alunos a se adaptarem a nova forma de realização de exercícios físicos - em casa - com o fechamento de locais como academias, parques, quadras e praças [1].

Houve estudos que realizaram a observação longitudinal comparando a população de estudantes universitários e a prevalência de depressão antes e durante a pandemia, concluíram que indivíduos se tornaram mais sedentários em relação aos períodos acadêmicos analisados anteriormente a esse cenário [4]. Por sua vez, a depressão foi prevalente em alunos que não praticavam exercícios físicos durante a pandemia [4,6].

Dentro desse cenário, os estudantes, por estarem em um cenário de confinamento, reduziram o nível de atividade física e a exposição solar diária - o que contribuiu para o aumento dos sintomas depressivos e de estresse, aliados ao isolamento social [3].

\section{Comportamento}

O aumento da incidência de pensamentos negativos, raiva, conflitos interpessoais e preocupações relacionados ao cenário pandêmico foram todos relacionados positivamente a depressão [1]. Tais comportamentos irritáveis sugerem que tanto o humor, como também o estado mental, podem ter sido afetados intrinsecamente devido ao distanciamento social e à falta de atividades sociais normais dos estudantes durante o isolamento [1]. A realidade do comportamento mais solitário é estatisticamente comprovada em estudos, uma vez que foi demonstrado que estudantes universitários, após o início da pandemia, visitaram menos lugares $(p<0,01)[4]$.

Foi observado que, após 2 (duas) semanas de confinamento, o medo de risco próprio de adquirir a infecção aumentou $(p<0,01)$, o que aumentou os transtornos de saúde mental, como estresse, ansiedade e depressão [9]. Além disso, os estudantes que já possuíam transtornos psiquiátricos como a depressão, durante a quarentena, encontram-se em fatores diminutivos de adesão ao próprio tratamento, uma vez que vivenciaram um período de maior índice e grau de depressão, ansiedade, solidão, ansiedade, negação, sintomas póstraumáticos, violência doméstica, instabilidade econômica e social, pensamentos suicidas, frustração devido a paralisação das atividades curriculares e diárias, desespero e insônia $[2,7]$.

Em um estudo em 6 universidades, 89,7\% dos pacientes optaram por ficar em casa durante a pandemia.
Porém, a duração do isolamento, de modo geral, não acompanhou o fim do mesmo pelas políticas de saúde, sendo que: isolamento durante menos que 1 (uma) semana $(n=147), 1-2$ semanas $(n=242), 2-4$ semanas $(n=$ 1704) e mais que 4 (quatro) semanas, apenas $(n=136)$ [11]. A análise das amostras de universitários da Grécia resultou em $74,3 \%$ dos participantes com depressão, em um aumento horizontal com predomínio de depressão leve [14]. Já em outros estudos, escores moderados a extremamente graves de depressão ocorreram em 34,19\% dos pacientes [10].

Foi verificado que a percepção individual de risco de infecção alto ou muito alto teve correlação com sintomas depressivos $(p<0,001)$ e menores correlações com provável diagnóstico de depressão, automutilação e ideação suicida (correlação = 0,07 e 0,08, p<0,001) [13]. Já, por sua vez, a prevalência de depressão provável moderada a grave conforme o Questionário de Saúde do Paciente-9 (PHQ-9) e ideação suicida e automutilação foi de $14,8 \%$ e 12,9\%, respectivamente [13].

\section{Sexo}

Ocorreu o predomínio de depressão e de ansiedade em estudantes do sexo feminino em diversas amostras, variando entre $54 \%$ até por volta de $70 \%$ dos universitários $[4,13,14]$.

Mas, com relação a gravidade da depressão, os casos em que homens apresentaram sintomas depressivos, estes eram mais elevados do que nas mulheres [6].

\section{Sono}

Sabe-se que o sono é um aspecto muito importante no quesito saúde. Durante a pandemia, houve um declínio na qualidade do mesmo, principalmente em indivíduos que aderiram ao isolamento $[3,8,14]$. Além disso também foi relatado em estudo que a quantidade de sono aumentou em $66,3 \%$ dos participantes [14].

É importante citar que para avaliar a qualidade de sono utilizou-se o Índice de qualidade do sono de Pittsburg (PSQI), teste o qual utiliza vários quesitos - quantas horas as pessoas ficam na cama, a que horas iam dormir e a que horas acordavam de manhã - dessa forma, foi possível perceber que a alteração na qualidade do sono foi mais forte em participante com alto escore DASS - 21 (maiores sintomas de depressão, ansiedade e estresse) principalmente em alunos [3]. Ademais, foi notado que os estudantes atrasaram seu tempo de despertar em aproximadamente 45 minutos [3]. 


\section{Tempo de tela}

A maioria dos participantes dos estudos ficaram mais horas no uso de aparelhagem eletrônica. Ademais, foi constatado que houve relação com elevação nos riscos de depressão, além de, afetação da visão e ainda potencial em provocar ganho de peso e favorecer o risco cardiovascular, devido a adoção de um estilo de vida sedentário [1]. Inclusive, o tempo de tela alargou-se drasticamente, em especial, antes de dormir, favorecendo uma qualidade de sono inferior [3].
Outra questão importante faz jus às diversas notícias improcedentes disseminadas pelos dispositivos midiáticos que contribuíram para o surgimento de problemas relacionados a saúde mental das pessoas, como a depressão e a ansiedade $[2,4]$.

\section{DISCUSSÃO}

A seguir, o esquema mostra os principais fatores relacionados à quarentena, ao cenário pandêmico e aos níveis de depressão em estudantes universitários.
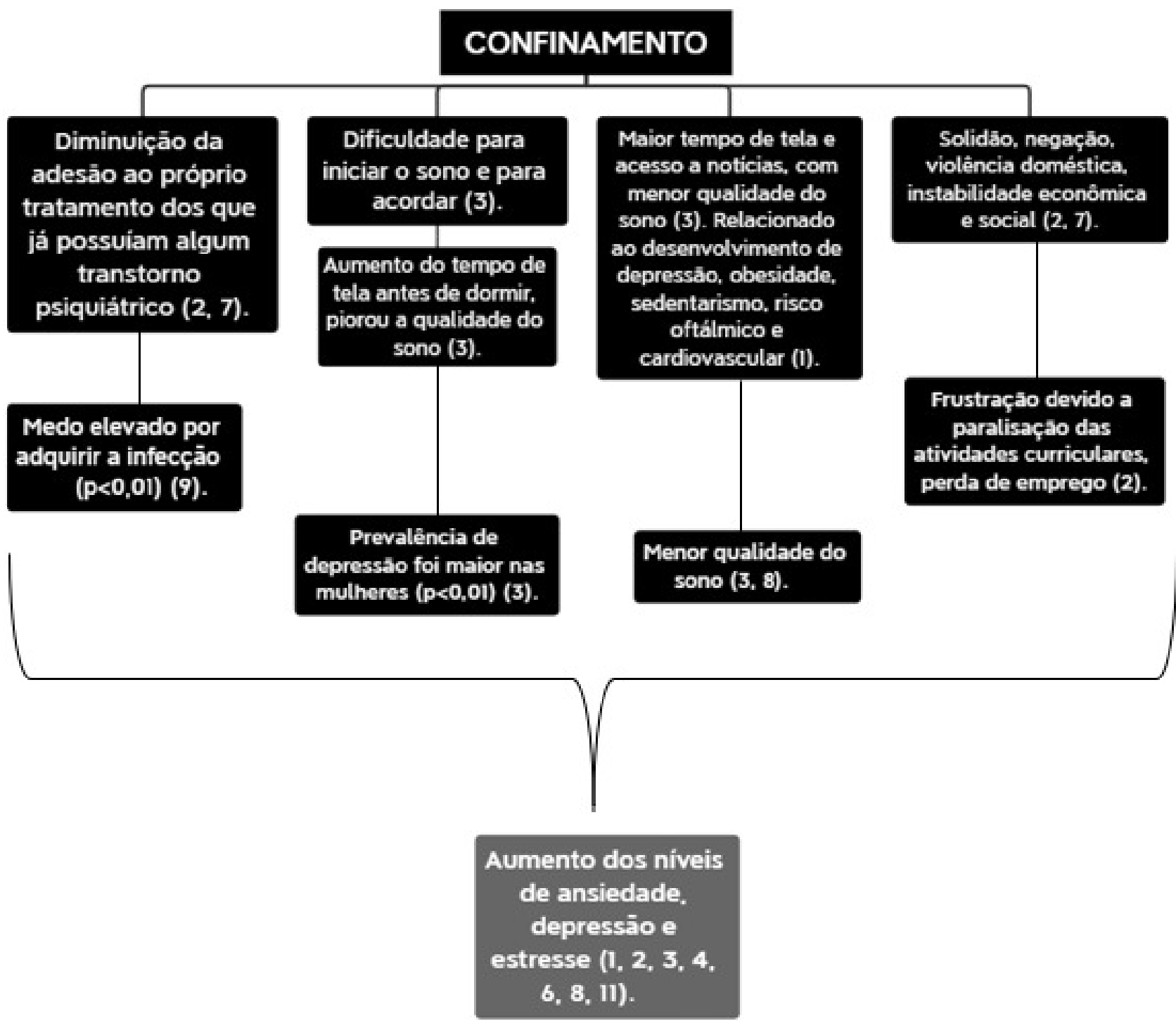

Figura 2. Elaborada pelos autores. 
Os universitários apresentam maiores níveis de depressão em comparação com outras populações - como por exemplo, alunos de mestrado e trabalhadores. $(p<0,05)$ [10]. A pandemia e o fechamento das universidades afetaram intrinsecamente os quadros depressivos de estudantes, principalmente por estarem longe de casa, inseguros quanto ao futuro, com medo de infecção própria e de conhecidos [2,9]. Desse modo, eles se mostraram, nas pesquisas, os mais vulneráveis aos sintomas de depressão e ansiedade relacionados ao estresse [1]. Em especial, os estudantes do curso de medicina mostraram-se com as maiores pontuações nos níveis de ansiedade, depressão e estresse. $(p=0,001)[7]$.

Aliado a esse cenário, notou-se ainda um atraso nas matérias e nas atividades curriculares, como também do desempenho em geral $(p=0,02)$, bem como, maior preocupação financeira. $(p=0,04)(6)$. Fato a se destacar é que, embora o fato seja ainda controverso, os maiores graus de depressão parecem se encontrar nos períodos mais avançados do curso superior $(P<0,001)[4,9]$.

Grupos de estudantes com e sem Coronavírus foram comparados e mostraram que os sintomas de depressão são preponderantes nos infectados $(p<0,001)$ [5]. Esse fato pode estar relacionado com o neurotropismo devido a infecção por SARS-CoV-2, aliados com o estresse póstraumático de uma patologia desconhecida [1].

Além disso, a prática de exercícios físicos foi um dos principais fatores impactados na pandemia, apesar da sua importância para a imunidade, saúde física e mental [8]. Com o cenário pandêmico, foram necessárias várias adaptações para essa prática, já que, a mesma impossibilitou a abertura de academias e centros esportivos sociais, sendo assim, muitos estudantes interromperam a prática, o que elevou a taxa de sedentarismo e a depressão foi prevalente em alunos que não praticam exercícios durante a pandemia $[4,6]$. Aliado ao menor nível de atividade física, o confinamento, o isolamento $(p<0,01)$ e a menor exposição solar diária resultaram no aumento de sintomas depressivos relacionados ao estresse e a ansiedade. [3,4].

O gráfico abaixo explana o impacto da pandemia pela COVID-19 nos níveis de depressão e sedentarismo em estudantes universitários no decorrer das semanas letivas.

\section{Depressão e Sedentarismo}

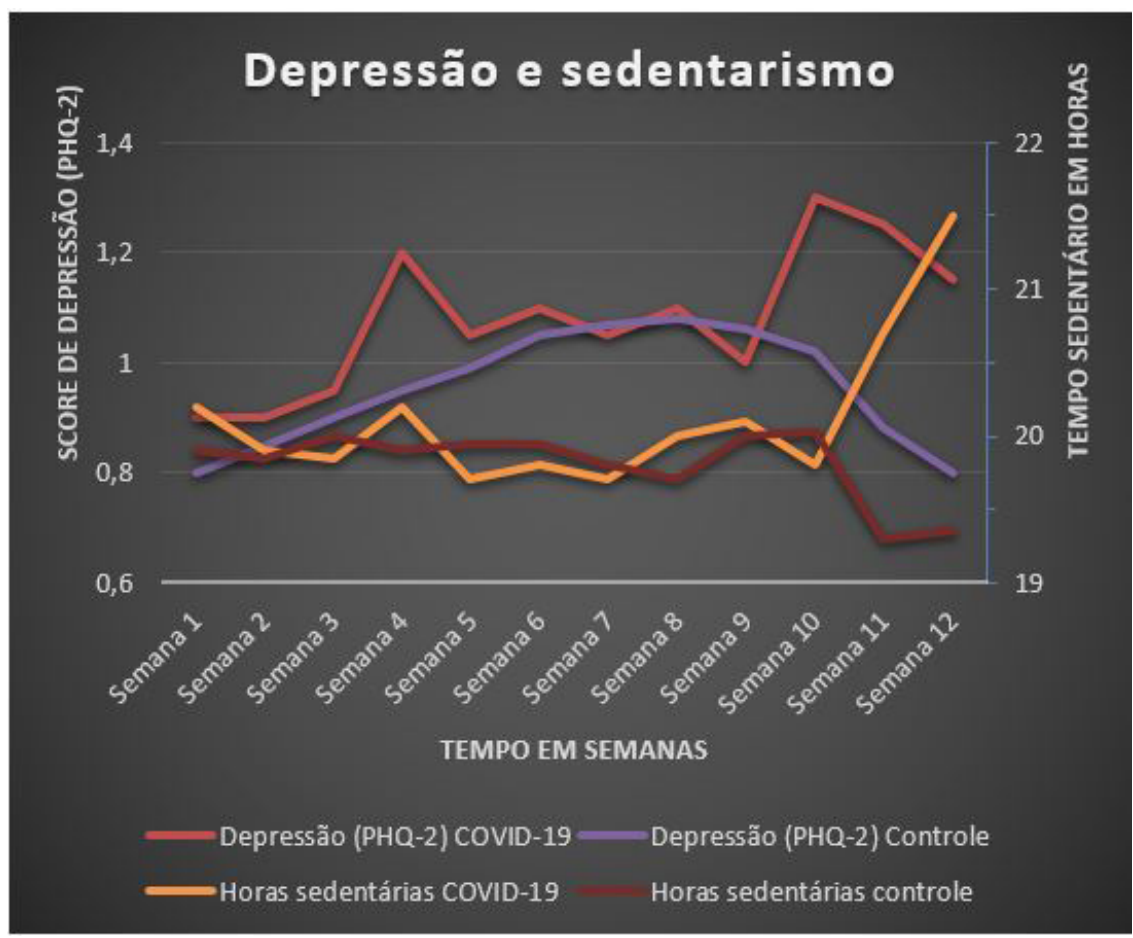

Gráfico 1. Demonstra níveis de depressão (de acordo com a escala pelo Questionário de Saúde do Paciente-2 (PHQ-2) [15] e sedentarismo em duas populações de universitários em diferentes períodos: antes e durante o COVID-19. A semana 1 representa o primeiro dia do ano letivo das duas populações, a partir da semana 3, observou-se o primeiro caso de COVID-19 próximo a população em cenário de pandemia [4]. Na semana 8, ocorreu o fechamento da universidade; a semana 10 denota o início dos exames finais, e a partir da semana 11, a interrupção das aulas [4]. Elaborado pelos autores. 
Diante do exposto, é possível inferir que é fundamental a compreensão do comportamento psicológico dos estudantes universitários - futuros profissionais e integrantes do mercado de trabalho. Para que, dessa forma, em futuros eventos pandêmicos, com o amparo da pesquisa, tenha-se o preparo do setor da saúde para a intervenção e a prevenção com relação a depressão e ansiedade na sociedade. Apesar extrema importância do tema, ainda há muito trabalho a ser feito. São necessários mais estudos longitudinais que comparem as populações antes e durante a pandemia uma vez que, apenas dois desse tipo foram encontrados em nossas bases de dados. Assim como, estudos com propostas de resolução e intervenção ao problema não foram encontrados. Somente com o maior sustento pela pesquisa científica seria possível a real visão dos diferentes padrões de comportamento e do real impacto - tanto qualitativo quanto quantitativo - que o cenário pandêmico causou na população e quais são as comprovadas respostas intervencionistas eficazes.

\section{Agradecimentos}

À professora orientadora Fernanda Liboni Cavicchioli, que, através de seus conselhos e ensinamentos, possibilitou a conclusão deste trabalho.

À amizade verdadeira conquistada na universidade, que nos proporciona momentos enriquecedores e de crescimento mútuo - tanto como seres humanos como também no âmbito profissional. Graças a ela, esses autores alcançaram a realização desse trabalho.

À Pontifícia Universidade Católica do Paraná por todo o suporte e apoio necessário.

\section{REFERÊNCIAS}

1. Xiao H, Shu W, Li M, Li Z, Tao F, Wu X, et al. Social distancing among medical students during the 2019 coronavirus disease pandemic in china: Disease awareness, anxiety disorder, depression, and behavioral activities. Int J Environ Res Public Health. 2020; 17 (14): 5047.. http:// dx.org/10.3390/ijerph17145047

2. Khan KS, Mamun MA, Griffiths MD, Ullah I. The Mental Health Impact of the COVID-19 Pandemic Across Different Cohorts. I Int J Environ Res Public Health. 2020; 1(1): 1-7. http://dx.org/10.1007 / s11469-020-00367-0

3. Cellini N, Canale N, Mioni G, Costa S. Mudanças no padrão de sono, senso de tempo e uso de mídia digital durante o bloqueio COVID-19 na Itália. J Sleep Res. 2020; 29 (4): e13074. http://dx.org/10.1111 / jsr.13074

4. Huckins JF, daSilva AW, Wang W, Hedlund E, Rogers C, Nepal SK, et al. Mental Health and Behavior of College Students During the Early Phases of the COVID-19 Pandemic: Longitudinal Smartphone and Ecological Momentary Assessment Study. J Med Internet Res. 2020 Jun 17;22(6):e20185. http://dx.org/10.2196/20185

5. Guo Q, Zheng Y, Shi J, Wang J, Li G, Li C, et al. Immediate psychological distress in quarantined patients with COVID-19 and its association with peripheral inflammation: A mixed-method study. Brain Behav Immun. 2020 Aug; 88:17-27. http://dx.org/10.1016/j. bbi.2020.05.038

6. Akhtarul IM, Barna SD, Raihan H, Nafiul AKM, Tanvir HM. Depression and anxiety among university students during the COVID-19 pandemic in Bangladesh: A webbased cross-sectional survey. PLoS ONE. 2020.

7. Vahedian-Azimi A, Moayed MS, Rahimibashar F, Shojaei S, Ashtari S, Pourhoseingholi MA. Comparison of the severity of psychological distress among four groups of an Iranian population regarding COVID-19 pandemic. BMC Psychiatry. 2020.

8. Dominski FH, Brandt R. Do the benefits of exercise in indoor and outdoor environments during the COVID-19 pandemic outweigh the risks of infection? Sport Sciences for Health. 2020.

9. Li HY, Cao H, Leung DYP, Mak YW. The psychological impacts of a covid-19 outbreak on college students in China: A longitudinal study. Int J Environ Res Public Health. 2020.

10. Odriozola-González P, Planchuelo-Gómez Á, Irurtia MJ, de Luis- García R. Psychological effects of the COVID-19 outbreak and lockdown among students and workers of a Spanish university. Psychiatry Res. 2020; 290:113108. http://dx.org/10.1016/j.psychres.2020.113108

11. Tang W, Hu T, Hu B, Jin C, Wang G, Xie C, et al. Prevalence and correlates of PTSD and depressive symptoms one month after the outbreak of the COVID-19 epidemic in a sample of home-quarantined Chinese university students. J Affect Disord. 2020; 274: 1-7. http:// 
dx.org/10.1016/j.jad.2020.05.0092020

12. Zhang Y, Cao X, Wang P, Wang G, Lei G, Shou Z, et al. Emotional "inflection point" in public health emergencies with the 2019 new coronavirus pneumonia (NCP) in China. J Affect Disord. 2020; 276: 797-803. http:// dx.org/10.1016/j.jad.2020.07.097

13. Xin M, Luo S, She R, Yu Y, Li L, Wang S, et al. Negative cognitive and psychological correlates of mandatory quarantine during the initial COVID-19 outbreak in China. Am Psychol. 2020 Jul-Aug;75(5):607-617. http:// dx.org/10.1037/amp0000692

14. Kaparounaki CK, Patsali ME, Mousa DP V, Papadopoulou EVK, Papadopoulou KKK, Fountoulakis KN. University students' mental health amidst the COVID-19 quarantine in Greece. Psychiatry Research. 2020. http:// dx.org/10.1016/ j.psychres.2020.113298 